\title{
VAC_21 - Development of influenza virus expressing the antigenic portion of SARS-Cov-2 S protein as a vaccine to prevent Covid19 and flu
}

Júlia T. Castro ${ }^{*}$; Gabriela de Assis Burle Caldas ${ }^{1}$; Ana Paula de Faria Gonçalves²; Daniel Doro 3 ; Kimberly Freitas Cardoso ${ }^{3}$; Santuza Maria Ribeiro Teixeira ${ }^{2}$; Alexandre de Magalhães Vieira Machado ${ }^{3}$; Ricardo Tostes Gazzinelli ${ }^{3}$.

${ }^{1}$ CT-Vacinas;

${ }^{2}$ UFMG - Universidade Federal de Minas Gerais;

${ }^{3}$ Fiocruz/CPqRR.

Introduction: Seasonal influenza viruses infect $5-15 \%$ of the human population each year, resulting in approximately 500,000 deaths worldwide. Influenza A viruses have two glycoproteins anchored on the viral envelope: haemagglutinin (HA) and neuraminidase (NA). The SARS-CoV-2 is a $\beta$-coronavirus that was discovered in December 2019 in Wuhan, China. SARS-CoV-2 is now responsible for an ongoing outbreak of atypical pneumonia that has affected people worldwide. Coronavirus spike (S) glycoprotein promotes entry into cells and comprises two functional subunits responsible for binding the host cell receptor: S1 subunit, which contains the receptor-binding domain (RBD) and S2 subunit, responsible for fusion between the viral and cellular membranes. As the $\mathrm{S}$ glycoprotein is surface-exposed and mediates entry into host cells, it is the main target of neutralizing antibodies upon infection and is the focus of therapeutics and vaccine design.

Objective: In this work we aimed to develop a bivalent vaccine against SARS-Cov-2 and seasonal flu using recombinant influenza virus with an impaired capacity to multiply.

Methodology: To do so we utilized eight plasmids to driven reverse genetics to generate a recombinant influenza virus carrying only the first 169 and the last 178 nucleotides of NA sequence. We used two different portions of S protein that are known to be immunogenic (RBD and RBD-SD1) and incorporated inside NA sequence to allow expression of this protein in the surface of the virus (169RBD and 169RBDSD1). In parallel we also generated a recombinant influenza virus in which the expression of the RBDSD1 domain of S protein is secreted in the cell (166RBD-SD1). Following Balb/c or C57BL/6 mice were immunized with two doses containing 105 PFU of 169RBD, 169RBD-SD1 or 166RBD-SD1 recombinant virus. To evaluate the immune response elicited by vaccination, we investigated the presence of specific IgG antibodies in mice sera and the production of IFN-g by splenocytes stimulated with recombinant RBD protein or RBD peptides.

Results: Immunofluorescence assays using antibodies against RBD and HA confirmed the generation of the recombinant influenza virus expressing SARS-CoV-2 RBD and RBD-SD1 domains. Immunization assays demonstrated that mice immunized with 169RBD, 169RBD-SD1 and 166RBD-SD1 recombinat virus produced only timid levels of anti-RBD IgG antibodies but high levels of anti-HA antibodies. Splenocytes from immunized mice stimulated with RBD or peptides from RBD protein were able to generate strikingly high levels of IFN-g, detected by ELISA and ELISPOT.

Conclusion: Immunization of mice with recombinant influenza virus expressing RBD and RBD-SD1 generated low levels of IgG antibodies but induced high levels of IFN-g. We are currently evaluating if immunization with the recombinant influenza virus expressing RBD and RBD-SD1 are capable to protect mice against a challenged with SARS-Cov-2 virus.

Keywords: SARS-Cov-2; Influenza virus; Vaccine 